\title{
ORIENTAÇÃO PARA MERCADO E INTERFACE FUNCIONAL: EVIDÊNCIAS EM PROJETOS DE DESENVOLVIMENTO DE NOVOS PRODUTOS
}

\author{
Roberto Sbragia \\ Doutor em Administração pela Universidade de São Paulo - USP \\ Professor Titular da Faculdade de Economia, Administração e Contabilidade da Universidade de São \\ Paulo - FEA/USP \\ rsbragia@usp.br (Brasil)
}

\section{Manuella Oliveira Lima}

Mestre em Administração pela Universidade de São Paulo - USP

manuellaolima@gmail.com (Brasil)

\section{RESUMO}

A inovação é reconhecida como uma das atividades que mais contribuem para o crescimento e manutenção da competitividade da empresa e pode estar associada a fatores importantes a serem considerados para se atingir o sucesso. Um deles consiste em transformar os sinais de mercado em respostas adequadas, em forma de produtos e serviços novos ou melhorados, por exemplo. O principal objetivo deste artigo é examinar a relação entre orientação para o mercado e interface funcional no contexto do desenvolvimento de novos produtos. A proposição básica é a de que as empresas mais orientadas para o mercado precisam de melhor integração funcional em seus esforços para criar e desenvolver novos produtos que respondam adequadamente aos sinais de mercado. Para tanto, realizouse uma revisão bibliográfica, a partir da qual se extraíram as principais premissas e vertentes teóricas que serviram de base para o estudo. Os dados foram coletados por meio de um levantamento (survey), utilizando-se um questionário pré-testado e validado pelo cálculo do coeficiente alpha de Crombach. A pesquisa foi feita com participantes de projetos de desenvolvimento de novos produtos, em empresas consideradas inovadoras, com operações no Brasil. O critério de seleção da amostra foi intencional, por acessibilidade. A pesquisa contou com a participação de 21 entrevistados. Para análise dos dados, utilizaram-se estatísticas descritivas e correlações bivariadas, com o cálculo do coeficiente $\rho s$ (rho de Spearman). A análise dos resultados apontou que a maioria das empresas apresentou de moderados a altos graus de orientação para mercado e de interface funcional. As diversas correlações encontradas entre os indicadores das variáveis analisadas mostraram que, na maioria dos casos, organizações mais orientadas para mercado possuem, também, maior integração e confiança entre as áreas funcionais em atividades ligadas ao desenvolvimento de novos produtos, na visão dos participantes desse tipo de projetos.

Palavras-chave: Inovação; Gestão; Interface funcional; Projetos; Desenvolvimento de produtos. 


\section{INTRODUÇÃO}

As aceleradas mudanças no contexto global e o perfil cada vez mais exigente dos consumidores alavancam o processo de inovação nas empresas em direção à diferenciação de seus produtos, serviços e processos, seja de forma incremental, seja radical. Porém a ânsia de colocar novos produtos no mercado pode esbarrar em riscos, como a incerteza do sucesso e os custos de falhas no processo de inovação. Nesse sentido, estabelecer boas práticas para o desenvolvimento de novos produtos é um meio para otimizar o processo de inovação.

Sabe-se que as boas práticas para o sucesso das inovações podem ser desenvolvidas tanto no âmbito corporativo da organização, quanto no de execução do projeto (Littler, 1994). Corporativamente, uma das estratégias utilizadas pelas organizações para fortalecer o desenvolvimento de novos produtos e a melhoria nos processos é a orientação para mercado. Segundo Rabechini, Carvalho e Laurindo (2002, p. 30), "há pelo menos três variáveis a serem consideradas na gerência da inovação: informação, tempo e pessoas. As empresas que melhor gerenciam a inovação são aquelas que disponibilizam a informação certa para a pessoa certa na hora certa". Essa afirmação reflete o conceito de orientação para mercado, que consiste na captação de informações pertinentes às necessidades do consumidor e ao comportamento dos concorrentes, na disseminação dessas informações entre as funções internas da organização, e na resposta a essas informações recebidas de forma adequada. Em razão da relevância da integração funcional, como pré-requisito para que a informação captada no mercado seja disseminada de forma adequada na empresa, identificou-se uma lacuna de conhecimento nas relações entre orientação para mercado e a interface funcional, especialmente em processos de desenvolvimento de novos produtos.

De fato, tomando-se como base pesquisas preliminares, notou-se que o campo de orientação para mercado já foi bastante explorado na literatura. Algo semelhante ocorre com o da interface funcional, em que artigos nas áreas de estruturas organizacionais, comunicação e gerenciamento de equipes trazem informações relevantes sobre o tema, porém de forma bastante dispersa. Todavia não foram encontradas pesquisas dedicadas a entender a existência de relação entre orientação no contexto de desenvolvimento de novos produtos. Considerando-se essa lacuna e entendendo-se que, tanto a estratégia de orientação para mercado quanto a existência de interface funcional carregam em sua essência a importância do fluxo de informações como base para suas teorias, o objetivo do estudo aqui apresentado é analisar as possíveis relações entre orientação para mercado e interface funcional, no contexto de desenvolvimento de novos produtos.

Revista de Administração e Inovação, São Paulo, v. 10, n.3, p.184-207, jul./set. 2013. 
A seguir, serão apresentados os principais aspectos da fundamentação teórica do estudo. No capítulo 3, serão mencionados os pontos mais relevantes da abordagem metodológica utilizada para efeito da pesquisa de campo. No capítulo 4, os principais resultados do estudo e as discussões pertinentes às descobertas realizadas. No capítulo 5 , por fim, serão descritas as principais conclusões e limitações do trabalho ora empreendido.

\section{FUNDAMENTAÇÃO TEÓRICA}

\subsection{Inovação e Desenvolvimento de Novos Produtos}

Historicamente, o campo de estudo da inovação divide-se em duas áreas principais. A primeira, numa abordagem voltada para a economia, examina os diferentes padrões de inovação em diferentes países e setores, bem como a evolução de tecnologias e a propensão intrassetores de inovar. A segunda área de estudo em inovação segue uma tradição voltada para a organização, focando questões de nível micro, como o desenvolvimento de novos produtos (Brown \& Eisenhardt, 1995). Este artigo utilizará como base a segunda área de estudos de inovação, tratando das relações intrafirma.

Diante da pressão sobre as empresas para inovar, acaba surgindo uma série de iniciativas na direção da busca pela novidade. Porém cabe destacar que, para o processo de inovação estar completo, é preciso ir além do insight inicial. A concepção da ideia é apenas o primeiro passo do desenvolvimento da inovação, o que pode ser reforçado pela visão de Schumpeter (1982, p. 62), segundo o qual "enquanto não forem levadas à prática, as invenções são economicamente irrelevantes", ou seja, apenas a ideia não garante impactos econômicos para a organização. Segundo Bamber, Owens, Davies e Suleman (2002), novos produtos e seu desenvolvimento bem-sucedido podem ser a vida da companhia. Em virtude disso, a inovação deve ser amparada por atividades que garantam o desenvolvimento e a entrega ao mercado da ideia inicial, para que a busca pelo novo não se torne prejuízo ao invés de benefício para a organização.

Nesse sentido, para que haja inovações concretas, relevantes para os resultados da organização, é necessária a existência de uma intensa atividade inovadora. De acordo com as definições encontradas no Manual Oslo (OECD, 2005), há diferenças entre inovação e atividade inovadora. Segundo o referido manual, uma inovação é a implementação de um produto (bem ou serviço) ou processo novo, ou significativamente melhorado, um novo método de marketing, ou um novo método organizacional de práticas internas, de organização do local de trabalho, ou de relações externas. Já a atividade inovadora,

Revista de Administração e Inovação, São Paulo, v. 10, n.3, p.184-207, jul./set. 2013. 
segundo o manual, é composta por sete pontos principais, a saber: 1) pesquisa e desenvolvimento; 2) engenharia; 3) início da produção; 4) marketing de novos produtos; 5) aquisição de tecnologias intangíveis; 6) aquisição de tecnologias tangíveis e 7) design. Dentre as atividades que levam à inovação, Andreassi e Sbragia (2002, p. 23) destacam pesquisa e desenvolvimento como a mais clássica, visto que assume papel de destaque perante as demais, influenciando o processo de inovação ativamente e influenciando o estado da arte das novas tecnologias. Adicionalmente, para Zawislak (1995, p. 12), "uma inovação é uma nova combinação de conhecimentos para gerar um novo, porém um novo conhecimento que tenha valor de troca e não só valor de uso". Com isso, entende-se por inovação o resultado de uma série de atividades inovadoras, cujo objetivo final é criar valor adicional ao produto, serviço, processo ou estrutura da organização.

Diante do conceito de inovação, cabe ainda um esclarecimento. Inovar nem sempre significa romper totalmente com o que existia. Muitas vezes, entende-se inovação como uma quebra de paradigmas, porém nem sempre é necessária uma ruptura total dos conceitos existentes para inovar. Neely e Hii (1998) relacionam os tipos de inovação existentes e as dimensões que esta inovação pode assumir. Para Neely e Hii (1998), as inovações podem ser classificadas como radicais ou incrementais. As primeiras dão origem a algo totalmente novo e as segundas, como o próprio nome diz, apenas incrementam o produto, adicionando um algo mais, significativo o suficiente para ser considerado inovador. Os autores afirmam ainda que a inovação pode ocorrer dentro de três dimensões: inovação no produto, inovação nos processos e inovação na estrutura organizacional. Os tipos de inovação podem, portanto, coexistir em diversas dimensões na organização, fato que torna a atividade inovadora diversificada e mais complexa.

Independentemente dos níveis em que a inovação é desenvolvida na organização, entende-se que, para sua realização, desde a concepção até a comercialização, configura-se um projeto, pois trata-se de um empreendimento composto por etapas definidas, com começo, meio e fim (Diegel, 2002). Nesse sentido, o modelo Stage Gate ${ }^{\mathrm{TM}}$ representa as fases do projeto de desenvolvimento de novos produtos, composto por uma série de estágios, intercalados por alguns pontos de revisão das fases (gates). Os estágios classificados por Cooper (1995) são representados em forma de etapas do projeto, enquanto os pontos situados entre um e outro estágio são paradas para feedback do que foi feito, antes que se inicie a etapa seguinte. $\mathrm{O}$ referido modelo pode ser visualizado na Figura 01. 


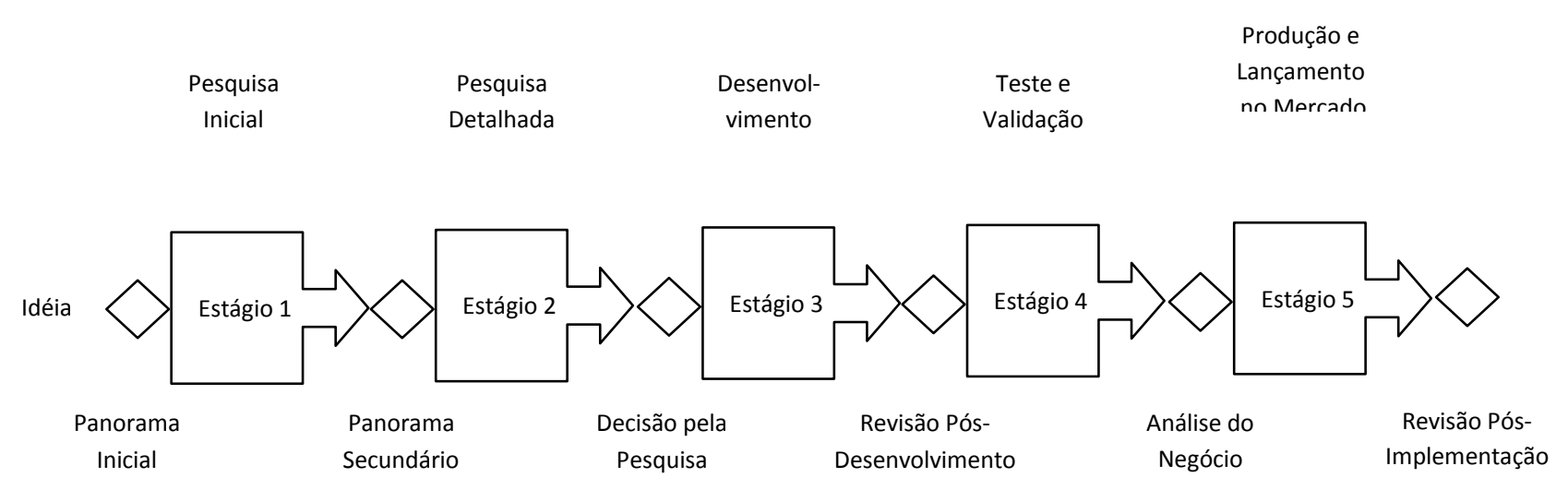

Figura 01. Processo de Desenvolvimento de Novos Produtos.

Fonte: Adaptado de Cooper (1995).

Segundo Bremser e Barsky (2004), o modelo Stage Gate provê um mecanismo que conecte o desenvolvimento de novas tecnologias às vendas, ao mercado e ao consumidor. Isso significa que a necessidade de feedbacks constantes, a cada nova fase do projeto, caracteriza maior interação entre as áreas responsáveis pela concepção do novo produto, ou serviço. Observando-se o modelo Stage Gate ${ }^{\mathrm{TM}}$, de Cooper (1995), entende-se que os projetos de desenvolvimento de novos produtos são formados por etapas integradas, cuja participação de diversas funções da empresa é requerida para o sucesso do resultado final, ou seja, da inovação.

Assim, cabe às organizações desenvolver estratégias que possam associar a valia das informações geradas, na organização e no mercado, ao desenvolvimento de novos produtos adequados aos desejos e necessidades dos consumidores, os quais podem tornar-se mais competitivos com relação aos produtos e serviços dos demais concorrentes do negócio. Uma das estratégias adotadas pelas organizações que seguem essa linha é a orientação para mercado. Mais detalhes sobre essa estratégia e sua aplicação para o sucesso das inovações poderão ser conhecidos no item seguinte desta fundamentação teórica.

\subsection{Orientação para Mercado}

Diante do exposto no tópico anterior, a orientação para mercado surge como alternativa para as empresas que desejam voltar-se para as necessidades do mercado e transformá-las em insumo para o aumento da capacidade competitiva das organizações. Em reforço a essa afirmação, Kara, Spillan, e Deshields (2005) afirmam que uma orientação para o mercado concede à empresa melhor entendimento de seus clientes, concorrentes e ambiente, o que, consequentemente, leva a um desempenho superior; esse desempenho pode ser representado, entre outros fatores, pela produção de inovações.

Revista de Administração e Inovação, São Paulo, v. 10, n.3, p.184-207, jul./set. 2013. 
Um estudo de Athuaene-Gima (1995), realizado com 275 empresas manufatureiras e de serviços, pesquisou a relação de orientação para mercado com a inovação. Os resultados da pesquisa reforçaram a relação positiva entre uma boa orientação para mercado e atividades bem-sucedidas na organização, como o desenvolvimento de novos produtos. Em complemento, o estudo de Vasquez, Santos e Álvares (2001) apontou para evidências empíricas de que as empresas mais orientadas para mercado tendem a ter melhor propensão para inovar e para comercializar um número maior de inovações que seus concorrentes. Seguindo a mesma linha, os estudos mais recentes de Verhees (2005), e Tajeddini, Trueman e Larsen (2006) também encontraram relações positivas entre orientação para mercado e inovação/propensão para inovar. Os resultados descritos acima reforçam, portanto, a importância da exploração da temática de orientação para mercado no contexto das inovações.

Para que se compreenda melhor o papel da orientação para mercado na organização e como se dá o seu funcionamento, torna-se necessário buscar e organizar neste estudo algumas definições do tema proposto. Day (1994) afirma que a orientação para mercado representa habilidades superiores de entender e satisfazer os consumidores. Dentro dessas condições, em uma das mais importantes referências sobre orientação para mercado da literatura, Shapiro (1988) afirma que para uma companhia ser orientada para mercado há três condições básicas: 1) todos os seus membros devem compreender seus mercados-alvo e as influências de compras envolvidas; 2) decisões táticas e estratégicas devem ser tomadas de forma interfuncional e interdivisional; 3) divisões e funções devem tomar decisões bem coordenadas e executálas com comprometimento.

No campo do estudo das teorias de orientação para mercado, duas abordagens distintas chamam atenção como as mais difundidas, a saber: 1) orientação para mercado como parte da cultura da organização; 2) orientação para mercado como característica comportamental. Na vertente cultural, os estudos de Narver e Slater (1990) e Slater e Narver (1994) são os mais representativos e serviram de inspiração para grande parte das pesquisas na área, que desejaram seguir essa abordagem. Os autores entendem a orientação para mercado como um fenômeno decorrente do ambiente e da cultura organizacional e afirmam que um negócio é orientado para mercado quando for sistemática e inteiramente comprometido com a criação contínua de valor superior para o consumidor. Na mesma linha de Narver e Slater, Deshpandé, Farley e Webster (1993) associaram o conceito de orientação para mercado à cultura organizacional. Em complemento a essa visão, Athuaene-Gima (1996) define orientação para mercado como um conjunto de atividades que refletem um grau de adoção da filosofia do conceito de marketing pela organização.

Revista de Administração e Inovação, São Paulo, v. 10, n.3, p.184-207, jul./set. 2013. 
Compondo a vertente comportamental, em trabalhos praticamente contemporâneos aos de Narver e Slater, Kholi e Jaworski (1990) e Kholi, Jaworski e Kumar (1993) definem orientação para mercado como a geração em toda a organização de inteligência de mercado, relativa às atuais e futuras necessidades dos clientes, disseminação da inteligência entre os departamentos, e a resposta de toda a organização a essa inteligência. O modelo proposto pelos autores é formado pelos seguintes constructos: 1) Geração de inteligência - refere-se à coleta e análise tanto das necessidades/preferências dos consumidores, quanto das forças que influenciam o desenvolvimento e refinamento dessas necessidades; 2) Disseminação de inteligência - consiste no processo e medida de troca de informação de mercado numa dada organização; 3) Reação à inteligência - diz respeito à ação tomada em resposta à inteligência gerada e disseminada.

Com base numa vasta revisão de literatura, os autores criaram uma escala, chamada por eles de MARKOR, que, após diversos testes, foi confirmada com 32 itens, divididos entre os três constructos apresentados. Num trabalho posterior, Kholi et al. (1993) validaram essa escala, por meio de um esforço investigativo mais avançado, o que deu ainda mais credibilidade ao trabalho e fez com que esse instrumento seja grande referência para trabalhos investigativos, com Narver e Slater (1990). Dada sua popularidade e aplicabilidade em diversos contextos organizacionais, a escala de Kholi e Jaworski foi utilizada e validada novamente em dois estudos mais recentes. Matsuno, Mentzer e Rentz (2000) promoveram um refinamento da escala e, no Brasil, a versão refinada de Matsuno et al. (2000) foi utilizada por Müller Neto (2005) numa pesquisa voltada à compreensão da relação entre orientação para o mercado, inovação e desempenho da organização. Essa versão da escala foi traduzida para o português e adaptada por meio de entrevistas com executivos e por pré-testes, para que adquirisse características próprias ao entendimento dos brasileiros.

Com a apreciação das definições de orientação para mercado, nota-se que, em estudos importantes, como os de Narver e Slater (1990) e Shapiro (1988), a interface entre as funções e os diferentes agentes da organização é considerada fator relevante para uma organização orientada para mercado. Nesse sentido, resumidamente, pode-se afirmar que orientação para mercado diz respeito à captação, difusão e resposta adequada à informação de mercado, processo para o qual a interface funcional contribui, exercendo papel integrador fundamental para o bom exercício da comunicação nas organizações.

Para compreender melhor a relevância da integração entre as funções da organização para a disseminação adequada das informações como resposta à orientação para o mercado, especialmente

Revista de Administração e Inovação, São Paulo, v. 10, n.3, p.184-207, jul./set. 2013. 
utilizada em projetos de inovação, no tópico seguinte apresentam-se estudos sobre interface funcional e o seu papel no processo de desenvolvimento de novos produtos.

\subsection{Interface Funcional}

Em diversos campos do estudo de administração, há o reconhecimento de que as funções devem estar interligadas para o melhor desempenho geral da organização. Para que se compreenda com clareza do que se trata a interface funcional, define-se função como "a agregação de atividades análogas, interdependentes, que se encadeiam num único campo especializado de trabalho..." (Cury, 2006, p. 192).

Nessa linha, especificamente no contexto do desenvolvimento de inovações, Moenaert e Souder (1990) ressaltam que, pelo fato de a inovação tecnológica ser uma sequência de atividades informaçãoprocessamento, ela requer a contribuição (inputs) de vários membros, de diferentes funções, desempenhando diversos papéis. Nesse sentido, o modelo apresentado por Purdon (1996) ajuda a entender como se daria a integração entre as principais funções existentes no desenvolvimento de novos produtos. $\mathrm{O}$ autor traz o conceito de campo de trabalho que, segundo ele, é a arena de atividade e geração contínua do conhecimento, na qual um papel organizacional é inicialmente estabelecido, o domínio pode ser desenvolvido e a criatividade emerge. Entende-se, com isso, que o campo de trabalho se forma a partir da interação das funções organizacionais e essa interação contribui para o processo de desenvolvimento de inovações. A Figura 02 mostra a interdependência entre as funções da organização para o desenvolvimento da inovação.

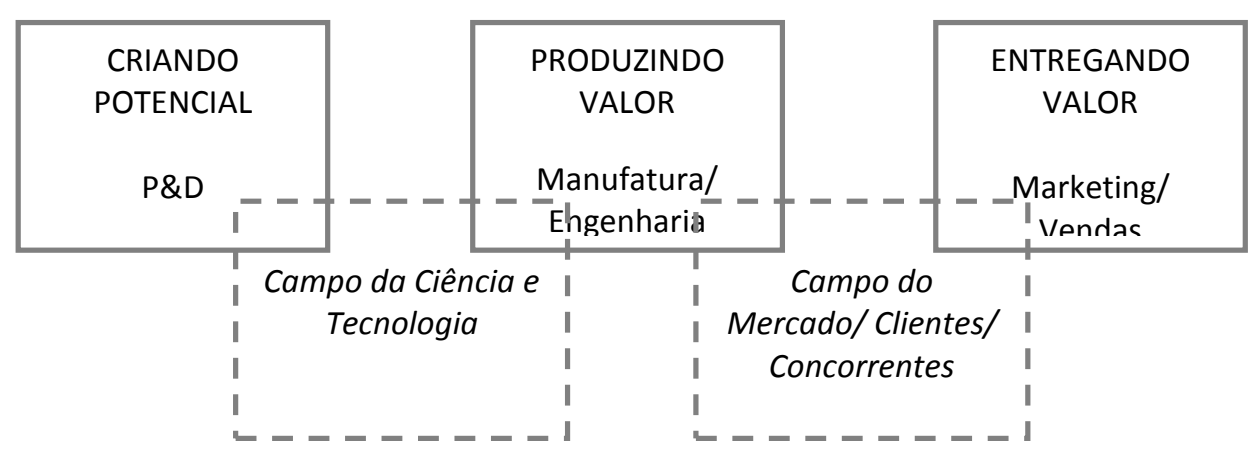

Figura 02. Interação entre as funções da organização na produção de inovação.

Fonte: Adaptado de Purdon (1996, p. 49). 
Observa-se na Figura 02 a interdependência entre as áreas funcionais que mais contribuem para a concepção e o desenvolvimento de inovações. Pelo modelo, pode-se perceber que a ligação entre P\&D e Engenharia forma o campo de Ciência e Tecnologia, enquanto a ligação entre Engenharia e Marketing/Vendas forma o campo de Mercado, Clientes e Concorrentes. Sendo assim, caso haja falha em um dos campos supracitados, a função de desenvolvimento de novos produtos estará prejudicada, devido à quebra de sinergia entre as funções da organização. Diante do exposto, nota-se que a tentativa de se estabelecer um relacionamento interfuncional pode resultar na geração de conflitos na organização. Essa crença é reforçada pela afirmativa de Buss (2002, p. 19), segundo a qual “o conflito interdepartamental é o grande 'efeito colateral' da interação interfuncional. Os fluxos de transação, ao mesmo tempo que aproximam e integram as funções, geram conflitos e barreiras que, por sua vez, dificultam o bom andamento dos fluxos”. Acredita-se, portanto, que os fenômenos negativos surgidos com a tentativa de se integrarem funções podem interferir na produção inovadora da organização.

Entre os estudos sobre interface funcional, boa parte dos artigos e teses encontrados enfatiza a relação entre Pesquisa e Desenvolvimento (P\&D) e Marketing. Em suas pesquisas, Moenaert e Souder (1990a) e Moenaert, Souder, Meyer, e Deschoolmeester (1994) afirmam que o sucesso das inovações tecnológicas requer contribuições tanto técnicas quanto de mercado e, com isso, enfatizam a importância da relação entre P\&D e Marketing. Gupta (1984) foi um dos pioneiros nas pesquisas envolvendo a interface P\&D-Marketing. Essa integração foi avaliada nesse estudo de duas perspectivas: necessidade percebida da integração e grau de integração alcançado. O estudo mostrou que, quanto mais arriscado for o desenvolvimento do produto e maior a incerteza ambiental percebida, maior será também a necessidade de integração entre P\&D e Marketing. O modelo de Song e Thieme (2006), por sua vez, tratando da interface P\&D-Marketing, carrega fortes características do modelo de Gupta (1984), mesmo tendo surgido vinte e dois anos depois, e destaca a importância do fluxo de informações para o sucesso do projeto de inovação.

Ainda no escopo dos estudos da relação entre P\&D e Marketing no campo das inovações, encontrou-se o estudo de García, Sanzo e Trespalacios (2008). Esse estudo é recente, bem fundamentado e buscou investigar a interface entre $\mathrm{P} \& \mathrm{D}$ e Marketing durante um processo de desenvolvimento de novos produtos. Um aspecto interessante desse estudo é a composição do constructo de interface funcional. Segundo os autores, a interface funcional é composta pela integração entre as funções e influenciada pela confiança existente entre os pares nessas duas áreas. Os resultados do estudo de García et al. (2008) apontaram para uma relação positiva entre confiança e integração entre as funções o que, em outras

Revista de Administração e Inovação, São Paulo, v. 10, n.3, p.184-207, jul./set. 2013. 
palavras, significa que quanto mais confiantes as pessoas estão, mais facilmente se dá a relação entre as funções da organização.

$\mathrm{Na}$ literatura estudada, encontraram-se, também, pesquisas relativas à interface marketingmanufatura, especificamente. Num estudo pioneiro, Shapiro (1977) ressaltou a importância da interface marketing e manufatura e questionou a possibilidade de essas funções coexistirem. Em complemento, Calantone, Dröge e Vickery (2002) concluem que quanto mais marketing conhece e quanto mais está apto a se comunicar com a manufatura, melhores serão as relações funcionais, maior será a utilidade da relação e menor o potencial de conflito. Foram também encontrados trabalhos sobre a interface marketing e outras funções da organização. Ruekert e Walker (1987) destacam que cada atividade de uma função afeta as atividades das outras envolvidas no processo. Isso ocorre por meio dos fluxos de comunicação, de recursos, de trabalho e de assistência e gera uma rede de interdependências entre as áreas.

No campo da relação de P\&D com as demais áreas da organização, foi encontrado o estudo de Kruglianskas (1981). O autor destaca a importância das interações para a eficácia do centro de P\&D e traz uma interessante definição para interação, a qual considera como "fluxos entre as unidades organizacionais e o centro de P\&D, constituídos por elementos tangíveis e intangíveis" (Kruglianskas, 1981, p. 74). Como reforço para a importância do estudo da interface entre P\&D e as demais áreas da organização, cabe destacar a citação de Roussel, Saad e Bohlin (1991, p. 17), segundo os quais “administrar estrategicamente P\&D significa, antes e acima de tudo, integrá-la na tecnologia e na estratégia de negócio. Depois, administrar o processo de P\&D, incluindo amplamente as ligações com outras peças críticas da estrutura corporativa".

Diante do exposto, chega-se a um ponto ainda não tocado neste artigo: a importância da gestão dos mecanismos de integração em projetos, especialmente no desenvolvimento de novos produtos. Nessa linha, Moenaert e Souder (1990a) afirmam que o valor da informação extrafuncional (advinda de outra função) está associado ao canal, à mensagem, à fonte e ao receptor, como na maioria dos processos de comunicação. Os autores afirmam, ainda, que esse valor pode variar dentro do processo de inovação, sendo afetado pelas características organizacionais, como formalização, centralização, clima e a estrutura do projeto.

Assim, passa a fazer sentido a compreensão de como gestão dos mecanismos de integração de projetos, no contexto do desenvolvimento de novos produtos, pode contribuir para que a informação advinda de mercado seja bem administrada no contexto interfuncional da organização. De fato, as correntes teóricas de desenvolvimento de novos produtos, orientação para mercado e interface funcional apresentam evidências da relevância de se realizar um estudo empírico em que se investigue uma suposta

Revista de Administração e Inovação, São Paulo, v. 10, n.3, p.184-207, jul./set. 2013. 
relação entre os dois últimos constructos. No capítulo a seguir apresentam-se os procedimentos metodológicos utilizados para efeito de campo.

\section{ABORDAGEM METODOLÓGICA}

O estudo ora desenvolvido possui natureza descritiva e correlacional. Quanto ao enfoque, pode ser considerado quantitativo, visto que foram utilizadas técnicas de análise estatística dos dados. O método do estudo pode ser classificado como não-experimental com aplicação de um corte transversal, ou seja, a realização da pesquisa numa única etapa (Sampieri, Collado \& Lucio, 2006). Para efeito de campo, foi escolhida a técnica de levantamento pela internet (e-survey).

Quanto ao modelo conceitual do estudo, as variáveis independentes pertencem ao constructo de Orientação para o Mercado de Kholi e Jaworski (1993), refinado por Matsuno et al. (2000) e adaptado e validado ao contexto brasileiro por Müller (2005). O constructo de orientação para mercado é composto por três variáveis, a saber: 1) Geração de Inteligência; 2) Disseminação da Inteligência; 3) Resposta à Inteligência. Já as variáveis dependentes pertencem ao constructo de Interface Funcional, desenvolvido e validado por García et al. (2008) e adaptado para esta pesquisa. O constructo da interface é composto por duas variáveis: 1) integração funcional e 2) confiança. No delineamento original do estudo (Lima, 2010), foram consideradas diversas outras variáveis, tais como porte, setor, estrutura organizacional da empresa, entre outras, as quais foram entendidas como moderadoras da relação supracitada. Todavia, em razão da limitação de espaço, os dados e as respectivas análises envolvendo essas variáveis serão inclusas neste artigo.

O universo da pesquisa foi composto por indivíduos empregados em empresas cujos nomes foram extraídos do ranking das 1250 empresas mundiais mais inovadoras do Department for Business, Enterprise \& Regulatory Reform [BEER] (2008). Essas pessoas participavam, ou já haviam participado, de projetos de desenvolvimento de novos produtos no período do levantamento. Pelo fato de não haver registro do número de funcionários envolvidos em projetos de novos produtos nas empresas consideradas, o universo foi classificado como infinito. O contato com as empresas foi estabelecido por ligações telefônicas e envio prévio de mensagem eletrônica, em que se forneciam os principais esclarecimentos sobre o estudo e um link para o questionário, que deveria ser disseminado entre os membros das equipes de novos projetos em suas organizações. Diante da dificuldade de acesso aos colaboradores para a

Revista de Administração e Inovação, São Paulo, v. 10, n.3, p.184-207, jul./set. 2013. 
aplicação da e-survey, definiu-se a amostra pelo critério de acessibilidade, ou conveniência (Sampieri et al., 2006).

Foram pesquisados 21 indivíduos, em 11 empresas diferentes, entre as quais: Alstom, Areva, BASF, Braskem, Embraer, Natura, Novartis, Pirelli, Sandvik, Siemens e Unilever. Os respondentes dividiram-se segundo esta composição: 66,7\% homens e 33,3\% mulheres, grande parte na faixa dos 26 a 45 anos e 36 a 45 anos, ambas representando 42,9\% dos casos estudados, enquanto os demais participantes das demais idades somaram 14,3\% da amostra. Como já era esperado, devido à natureza do tema deste estudo, as áreas em que os entrevistados mais se concentravam, no momento de sua realização, eram Pesquisa e Desenvolvimento (42,9\%), Marketing (19\%) e Gestão de Projetos (19\% dos respondentes). Acompanhando a lógica deste fenômeno, a função exercida pelos entrevistados dentro da organização que mais apareceu na caracterização da amostra foi Gerente do Projeto, para 33,3\% dos entrevistados. Em 28,6\% dos casos, os pesquisados eram membros de equipes de projetos, mas sem a função gerencial. Com relação ao tempo em que os entrevistados já participavam de projetos de desenvolvimento de novos produtos, 33,3\% responderam participar desse tipo de atividade de 3 a 5 anos, enquanto 23,8\% já participavam de projetos de novos produtos há mais de 10 anos. Entende-se, assim, que foi obtida, embora demasiadamente pequena, uma amostra equilibrada de respondentes para o estudo, evidenciando dados provenientes de diversos setores e perfis de participantes.

No questionário, fez-se uso de escalas nominais, para as etapas de caracterização da empresa e do entrevistado. Além disso, foram utilizadas também, para mensuração da orientação para mercado e interface funcional, escalas ordinais de concordância, com seis pontos, variando de Discordo Totalmente a Concordo Totalmente. A survey foi realizada por meio virtual. Para tanto, foi utilizado o software QuestionPro, específico para a elaboração e aplicação de questionários on-line. Após a tabulação e o tratamento dos dados, criou-se uma matriz no software de análise estatística SPSS (Statistic Package for Social Science). Para a verificação da confiabilidade da escala, após a realização do levantamento, foi calculado o coeficiente alpha de Crombach para as escalas de orientação para mercado e interface funcional. Os coeficientes alpha obtidos para as escalas foram todos acima de 0,7. Esse seria o mínimo aceitável para que a escala pudesse ser considerada confiável (Hair, Anderson, Tatham \& Black, 1998).

No processo de análise, calculou-se inicialmente a frequência absoluta e relativa para cada uma das variáveis dos constructos, tanto dependentes quanto independentes, com o objetivo de se traçar um panorama inicial dos dados obtidos. No campo das análises bivariadas, foram calculadas correlações entre cada indicador das variáveis de orientação para mercado e interface funcional. Para tal cálculo, utilizou-se o coeficiente de correlações posto-ordem de Spearman $\left(\rho_{\mathrm{s}}\right)$. Esse teste permite verificar se duas variáveis

Revista de Administração e Inovação, São Paulo, v. 10, n.3, p.184-207, jul./set. 2013. 
estão associadas positiva ou negativamente, ou seja, elas variam de -1 (correlação negativa perfeita) a +1 (correlação positiva perfeita); quanto mais perto desses valores, maior a correlação (Sampieri et al., 2006).

\section{RESULTADOS E DISCUSSÃO}

Neste tópico serão apresentados os principais resultados do estudo. Primeiramente, será feito um resumo das estatísticas descritivas das variáveis pesquisadas. Posteriormente, serão apontadas as principais evidências de relações entre orientação para mercado e interface funcional.

Quanto à análise descritiva, apresenta-se um resumo da distribuição das variáveis na Figura 03 e na Figura 04. Em ambas, os dados plotados apontam para um alto índice de orientação para mercado e de interface funcional, respectivamente.

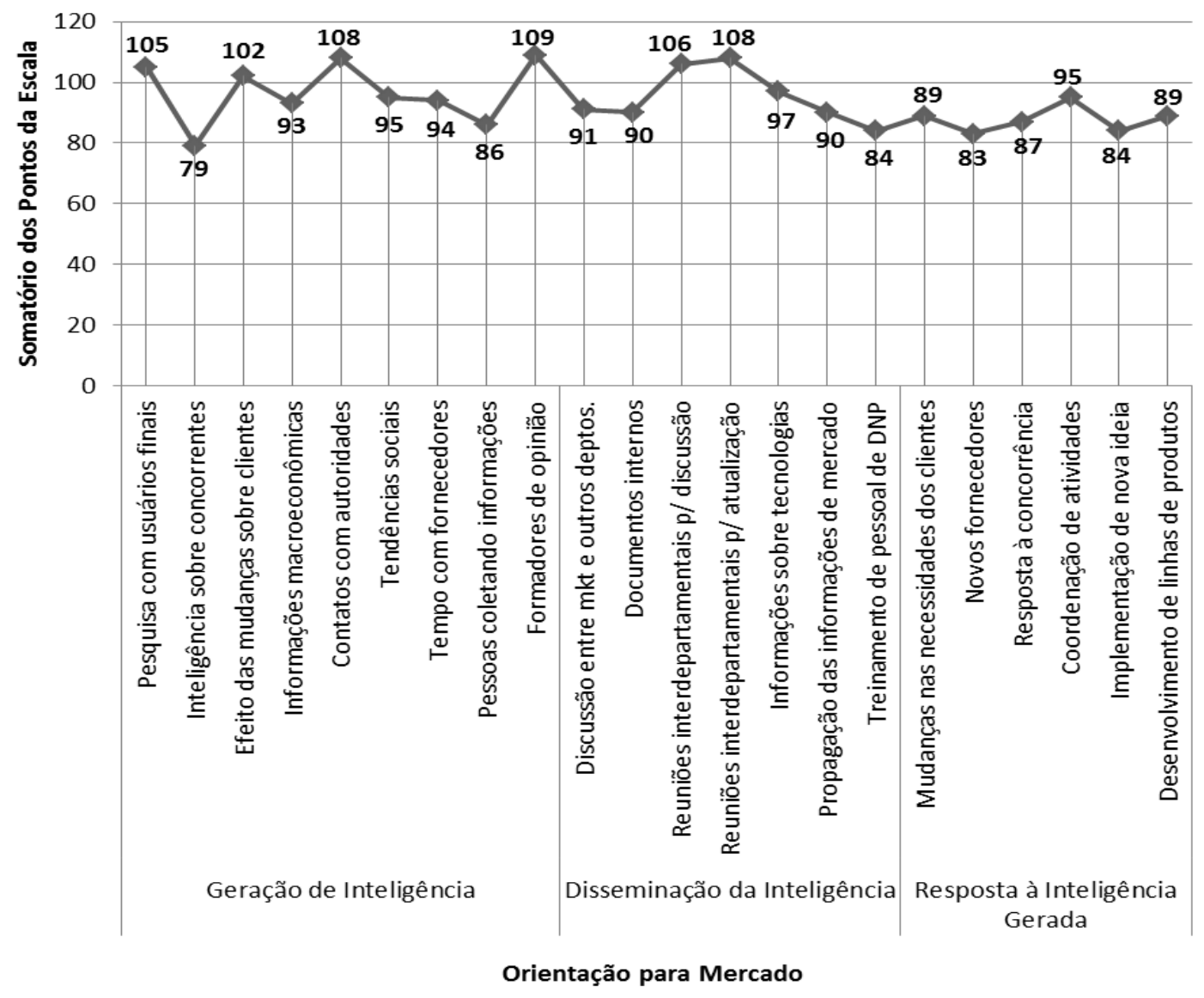

Figura 03. Distribuição dos dados para o constructo de Orientação para Mercado. Fonte: Elaborada pelos autores. 
Mais especificamente, na Figura 03 mostram-se os dados referentes a cada indicador do constructo de orientação para mercado, dividindo-o em: 1) Geração da Inteligência; 2) Disseminação da Inteligência e 3) Resposta à Inteligência. Nota-se na Figura que o primeiro grupo de indicadores apresenta os mais altos índices do constructo, com destaque para Pesquisa com Usuários Finais, Contato com Autoridades e Formadores de Opinião. Esses primeiros resultados, portanto, apontam para uma preponderância das atividades de geração de inteligência desenvolvidas por meio do contato pessoal. Ou seja, tanto nas pesquisas com usuários finais, no contato com autoridades, quanto nas pesquisas com formadores de opinião, entende-se que pode haver uma preocupação das equipes de projetos com o contato pessoal na busca de informações fidedignas sobre o que deseja o mercado. Ressalta-se ainda que o indicador com menor grau de manifestação entre as respostas da variável de geração da inteligência foi inteligência sobre os concorrentes. Provavelmente, esse fenômeno tenha ocorrido pelo fato de haver dificuldade, além da necessidade de altos investimentos, para se captarem informações sobre concorrentes no mercado.

Quanto à variável disseminação da inteligência, que representa a forma como as informações geradas no mercado são propagadas pelo projeto, os indicadores que apresentaram maior destaque foram: Reuniões Interdepartamentais para Discussão e Reuniões Interdepartamentais para Atualização. Novamente: comparando-se estes com os dos demais indicadores da variável, observa-se uma tendência à valorização dos contatos pessoais também para a disseminação da informação gerada. Em contrapartida, o treinamento de pessoal de desenvolvimento de novos produtos obteve a mais baixa pontuação entre os indicadores; isso pode indicar que, na visão dos pesquisados, essa forma de disseminação de informação esteja obsoleta, ou já não seja mais suficiente para satisfazer as necessidades das equipes de novos produtos.

Por fim, observa-se que a variável de resposta à inteligência obteve as menores pontuações no constructo de orientação para mercado. Com esse resultado entende-se que, na visão dos respondentes, há ainda dificuldade para se responder à informação gerada no mercado e trazida para dentro das equipes de novos produtos. De acordo com o desenho apresentado na Figura 03, pode-se entender que a resposta à inteligência mostra-se mais evidente em atividades internas, como é o caso da coordenação de atividades, mas deixa a desejar em processos que envolvam outputs, como a contratação de novos fornecedores ou a implementação de novas ideias no mercado.

Diante desse cenário, observa-se que as variáveis referentes à Orientação para o Mercado se comportam numa tendência decrescente, na qual a informação perde força do momento que sai do mercado e entra na organização e, lá dentro, precisa ser processada para gerar uma resposta. Nesse sentido, o processo obedece a um ciclo de geração, disseminação e resposta à informação que está sendo

Revista de Administração e Inovação, São Paulo, v. 10, n.3, p.184-207, jul./set. 2013. 
enfraquecido em alguns de seus elos, o que pode causar falhas no processo e resultar em projetos de novos produtos pouco compatíveis com a demanda do consumidor, ilustrando o famoso desentendimento interfuncional, que frequentemente dá origem a atrasos, retrabalho, conflitos, entre outros problemas.

No constructo de Interface Funcional, conforme Figura 04, as variáveis confiança e integração apresentaram também de moderados a altos valores para a soma das respostas. Os indicadores de confiança que apresentaram mais alta concordância por parte dos pesquisados foram Preocupação com os Objetivos e Sinceridade Mútua. Estes resultados, agregados a somas mais baixas para Expectativas da Equipe e Cumprimento das Promessas, podem sinalizar que, apensar de certa preocupação com os objetivos propostos para o projeto e com o bom convívio por meio da existência de sinceridade mútua, as expectativas das equipes não estão sendo totalmente satisfeitas e não está havendo um cumprimento das promessas feitas. Sendo assim, entende-se que a confiança, neste caso, é tratada como algo muito mais intangível (no campo das relações e das intenções) do que tangível (no campo do cumprimento das promessas e das satisfações reais das expectativas geradas).

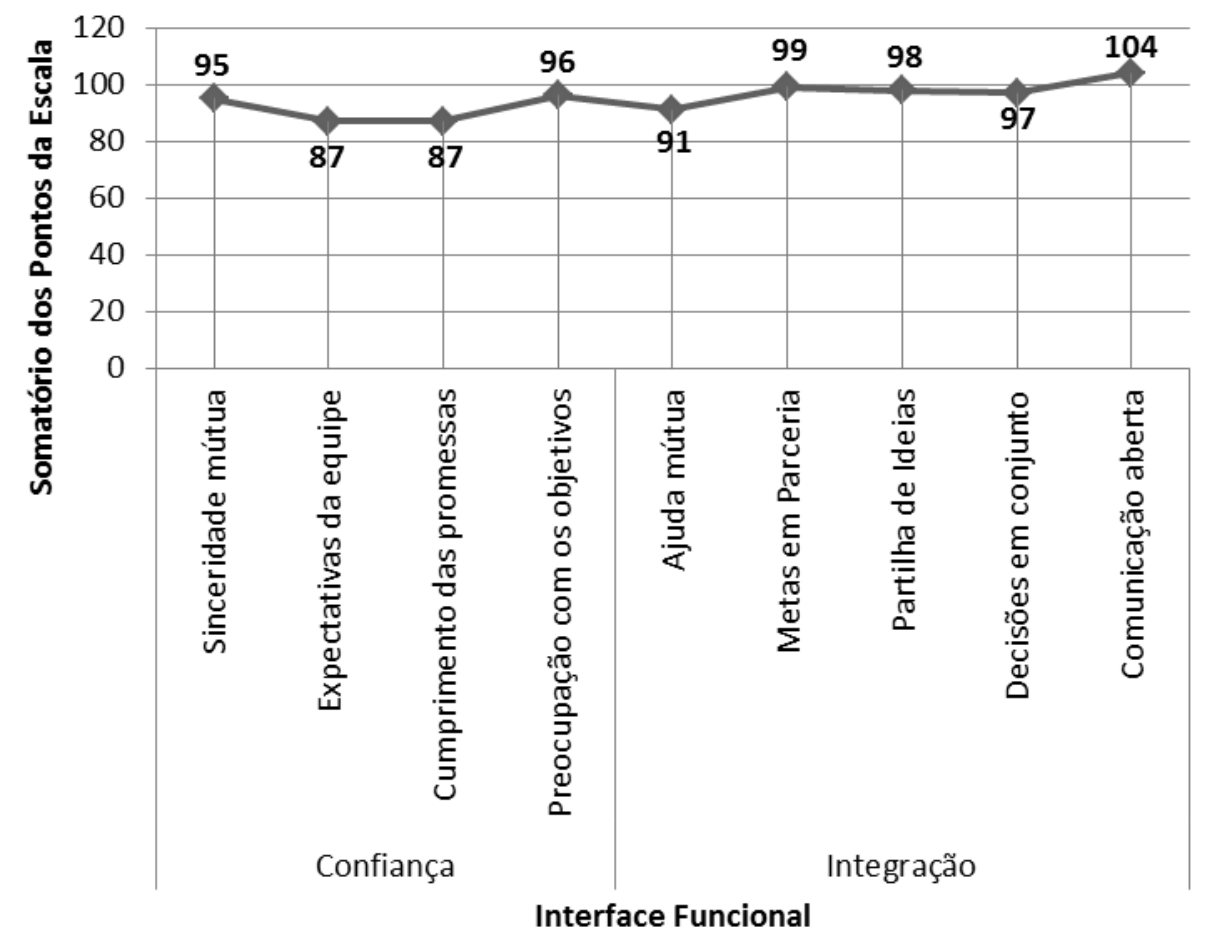

Figura 04. Distribuição dos dados para o constructo de Interface Funcional. Fonte: Elaborada pelos autores.

Nota-se ainda que o comportamento do gráfico na Figura 04 desenha um cenário de menor confiança despertada, porém de maior integração obtida entre as partes envolvidas nos projetos de desenvolvimento de novos produtos, pois os indicadores que representam atividades em parceria (à direita

Revista de Administração e Inovação, São Paulo, v. 10, n.3, p.184-207, jul./set. 2013. 
da Figura 04) apresentaram as maiores somas do constructo, especialmente no que diz respeito à comunicação aberta entre os membros do grupo e ao estabelecimento de metas em parceria. O comportamento do gráfico na Figura 04 pode apontar para uma tendência de as pessoas em equipes de novos produtos trabalharem integradas para o cumprimento de metas, mas não acreditarem tanto em fazerem parte de um grupo confiável.

Após a análise descritiva das variáveis, apresentam-se a seguir as correlações mais significativas encontradas entre os constructos de orientação para mercado e os de interface funcional. Na Tabela 01 apresentam-se os principais resultados encontrados, considerando-se a amostra total e níveis de significância menores ou iguais a 0,01 e 0,05 para o coeficiente de Spearman. Inicialmente, na correlação entre orientação para mercado e confiança, observa-se uma possível relação positiva entre os indicadores Pesquisa com Usuários Finais e Cumprimento de Promessas. O resultado pode sugerir que as equipes cumpram melhor suas promessas quando a informação advinda do mercado envolve pesquisa com usuários finais. Tal cenário pode apontar, novamente, para o fato de que as equipes de projetos valorizam a pessoalidade das informações, ou seja, pode haver indícios de que quanto mais próximos dos usuários finais (consumidores) os membros das equipes de projetos se sentirem, maior será a confiança despertada e, portanto, melhores tenderão a ser os resultados finais obtidos.

Já nas correlações entre orientação para mercado e integração, merecem destaque as relações entre os indicadores orientação para mercado e ajuda mútua, partilha de ideias e comunicação aberta entre os membros das equipes de desenvolvimento de novos produtos. Na geração da inteligência, observa-se que o efeito das mudanças no ambiente de negócios sobre os clientes e, novamente, a presença dos formadores de opinião, estão correlacionados positivamente com comunicação aberta nas equipes de projetos. Ou seja, pelo panorama apresentado, pode-se entender que tende a haver uma comunicação mais aberta entre os membros das equipes de novos produtos, quando são observados os efeitos das mudanças do mercado sobre os clientes e quando a fonte de informação são indivíduos formadores de opinião em determinado meio onde se deseja implementar o novo produto.

Tabela 01 - Correlações entre Orientação para Mercado e Interface Funcional (n=21)

\begin{tabular}{|c|c|c|c|c|c|c|c|}
\hline & \multirow{2}{*}{$\begin{array}{c}\text { Confiança } \\
\text { Cumpri- } \\
\text { mento de } \\
\text { Promessas }\end{array}$} & \multicolumn{5}{|c|}{ Integração } \\
\hline & & & $\begin{array}{l}\text { Ajuda } \\
\text { Mútua }\end{array}$ & $\begin{array}{c}\text { Metas } \\
\text { em } \\
\text { Parceria }\end{array}$ & $\begin{array}{l}\text { Partilha } \\
\text { de Ideias }\end{array}$ & $\begin{array}{c}\text { Decisões } \\
\text { em } \\
\text { Conjunto }\end{array}$ & $\begin{array}{c}\text { Comunicação } \\
\text { Aberta }\end{array}$ \\
\hline \multirow{2}{*}{ 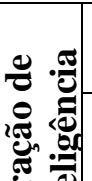 } & $\begin{array}{l}\text { Pesquisa com } \\
\text { usuários finais }\end{array}$ & $0,552 * *$ & & & & & \\
\hline & $\begin{array}{c}\text { Mudanças Sobre } \\
\text { Clientes }\end{array}$ & & $0,496^{*}$ & & & $0,527^{*}$ & $\mathbf{0 , 5 7 0} * *$ \\
\hline
\end{tabular}

Revista de Administração e Inovação, São Paulo, v. 10, n.3, p.184-207, jul./set. 2013. 


\begin{tabular}{|c|c|c|c|c|c|c|}
\hline & $\begin{array}{c}\text { Informações } \\
\text { Macroeconômicas }\end{array}$ & $0,537 *$ & & & & \\
\hline & $\begin{array}{l}\text { Tendências } \\
\text { Sociais }\end{array}$ & & & & $0,472^{*}$ & \\
\hline & $\begin{array}{l}\text { Tempo com } \\
\text { Fornecedores }\end{array}$ & & & $0,456^{*}$ & & $0,549 *$ \\
\hline & $\begin{array}{l}\text { Formadores de } \\
\text { Opinião }\end{array}$ & $0,661 * *$ & $0,451^{*}$ & $\mathbf{0 , 6 5 5} * *$ & & $\mathbf{0 , 5 6 1 * *}$ \\
\hline 莺 & $\begin{array}{c}\text { Discussão MKT- } \\
\text { Outros } \\
\text { Departamentos }\end{array}$ & & & & & $0,474 *$ \\
\hline (ָ) & $\begin{array}{l}\text { Reuniões } \\
\text { Interdep. } \\
\text { Atualização }\end{array}$ & & & $0,631 * *$ & & \\
\hline i్ & $\begin{array}{c}\text { Informações sobre } \\
\text { Tecnologias }\end{array}$ & $0,620 * *$ & & $0,687 * *$ & & $0,687 * *$ \\
\hline 吾 & $\begin{array}{l}\text { Propagação das } \\
\text { Informações de } \\
\text { Mercado }\end{array}$ & $0,508^{*}$ & & $0,457^{*}$ & & \\
\hline & $\begin{array}{l}\text { Treinamento de } \\
\text { Pessoal de DNP }\end{array}$ & & & & & $0,485^{*}$ \\
\hline & $\begin{array}{l}\text { Mudança nas } \\
\text { Necessidades }\end{array}$ & & & & & $0,640 * *$ \\
\hline 幽 & $\begin{array}{c}\text { Novos } \\
\text { Fornecedores }\end{array}$ & & & & $0,450 *$ & \\
\hline 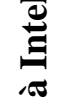 & $\begin{array}{l}\text { Coordenação de } \\
\text { Atividades }\end{array}$ & & & $0,437^{*}$ & & $0,672^{* *}$ \\
\hline 营 & $\begin{array}{l}\text { Implementação de } \\
\text { Nova Ideia }\end{array}$ & & $0,455^{*}$ & & $0,567 * *$ & \\
\hline$\stackrel{\overrightarrow{0}}{\tilde{e}}$ & $\begin{array}{l}\text { Desenvolvimento } \\
\text { das Linhas de } \\
\text { Produtos }\end{array}$ & & & & $0,511^{*}$ & \\
\hline
\end{tabular}

Fonte: Elaborada pelos autores.

* Nível de significância $\leq 0,05$.

** Nível de significância $\leq 0,01$.

No campo da disseminação das informações geradas, pode haver uma relação significativa entre reuniões interdepartamentais para atualização da equipe e partilha de ideias. Como era de se esperar, observando-se os resultados que indicaram a relevância das reuniões para a disseminação da inteligência (Figura 03), os pesquisados tendem a associar a realização de reuniões de atualização à possibilidade de partilhar ideias entre os membros da equipe. Novamente, destaca-se a importância do contato pessoal para a disseminação das informações geradas no ambiente de projetos de novos produtos como evidência nas análises realizadas.

Ainda em se tratando da relação entre disseminação da inteligência e integração (Tabela 01), cabe destacar as associações entre o compartilhamento de informações sobre tecnologias entre departamentos 
envolvidos nas equipes de projetos de novos produtos com ajuda mútua, partilha de ideias e comunicação aberta. Seguindo o mesmo raciocínio aplicado até agora, entende-se que quanto mais as informações sobre tecnologias forem partilhadas entre os departamentos, mais integrada tende a ficar a equipe de projetos de novos produtos.

Como se observou na revisão da literatura deste estudo, as áreas envolvidas em projetos possuem diversas especialidades e, consequentemente, dispõem de informações e tecnologias distintas, as quais devem ser compartilhadas no ambiente de projetos de novos produtos para que, a partir de uma integração maior, haja também a possibilidade de se responder mais eficazmente aos anseios do mercado.

Com relação à resposta às informações geradas e disseminadas pelas equipes de projeto versus integração (Tabela 01), observou-se que a atenção às mudanças nas necessidades dos clientes e a coordenação das atividades entre os departamentos envolvidos nos projetos associaram-se positivamente com comunicação aberta. Isso pode indicar que os respondentes associam uma resposta à inteligência gerada por meio destes dois indicadores (mudanças nas necessidades e coordenação das atividades) à abertura de comunicação dentro da equipe do projeto.

Encontrou-se, ainda, a correlação positiva entre implementação de uma nova ideia no desenvolvimento do novo produto e decisões em conjunto tomadas pelas equipes de projeto, o que pode significar que as novas ideias implementadas no decorrer de um projeto são fruto da sinergia entre os membros das equipes e sua capacidade de tomar decisões em conjunto. Nesse sentido, entende-se que, caso não haja consenso nem concordância entre a maioria, possivelmente não haverá também a implementação de novas ideias no projeto, que podem ser desperdiçadas, ocasionando respostas inadequadas ao mercado.

\section{CONCLUSÕES E CONSIDERACÇÕES FINAIS}

O objetivo deste trabalho foi analisar a relação entre a orientação para mercado e a interface funcional no contexto dos processos de desenvolvimento de novos produtos por empresas. A metodologia utilizada foi de natureza descritiva e correlacional e envolveu a realização de uma survey on-line, com participantes de projetos de desenvolvimento de novos produtos em organizações consideradas inovadoras de acordo com o ranking do BEER (2008). As variáveis envolvidas no estudo, trabalhadas na forma de constructos devidamente validados, foram Orientação para Mercado e Interface Funcional.

Revista de Administração e Inovação, São Paulo, v. 10, n.3, p.184-207, jul./set. 2013. 
Analisaram-se quantitativamente os dados, por meio de estatísticas descritivas e do coeficiente de correlações posto-ordem de Spearman $\left(\rho_{\mathrm{s}}\right)$, indicado para a análise de dados não paramétricos.

Como resultado, de forma geral, na visão dos participantes da pesquisa, encontrou-se uma alta manifestação de orientação para mercado e de interface entre as funções da organização. O resultado, em grande parte, está paralelo à teoria, em que estudos semelhantes, como o de Müller (2005), realizado no Brasil, também apontaram para altos graus de orientação para mercado entre os casos estudados.

Nas relações entre geração de inteligência e integração funcional, considerando-se as evidências em projetos de novos produtos, os resultados encontrados ratificam a importância dos formadores de opinião como fonte de informação para manter a equipe de projetos integrada. Nesse sentido, acredita-se que as informações advindas de formadores de opinião podem interessar a boa parte da equipe do projeto e promover a integração na equipe. O estudo permite supor ainda que, na opinião dos pesquisados, a manutenção da ajuda mútua e da comunicação aberta entre as áreas envolvidas nos projetos de novos produtos seja um fator relevante para a informação proveniente de áreas específicas da equipe ser disseminada entre as demais funções envolvidas nos projetos. Pode-se deduzir, com base nos resultados, que quanto mais bem disseminada for a informação dentro do projeto, mais os departamentos terão chance de ajudar uns aos outros, sendo possível, assim, desenvolver uma comunicação mais aberta.

Além disso, no campo da confiança entre as partes envolvidas no projeto, entende-se que a forma como a informação advinda do mercado é disseminada dentro do projeto pode fazer com que as expectativas da equipe pelas demais áreas do projeto sejam satisfeitas em maior ou menor graus. Por fim, o estudo permite entender que a satisfação da equipe envolvida no projeto varia de acordo com a forma pela qual as informações advindas do mercado são disseminadas entre as áreas funcionais da organização. Nesse sentido, quanto mais bem disseminadas as informações, mais satisfeita e confiante estará a equipe e, possivelmente, melhores serão as respostas geradas para as necessidades do projeto.

Como todo estudo, este artigo oferece uma contribuição para o fortalecimento dos alicerces da ciência. Porém cabe mencionar algumas limitações, que restringem o entendimento e inferência dos resultados obtidos. Assim, inicialmente, é preciso considerar que o estudo apresentado neste artigo teve como objetivo levantar possíveis relações entre as variáveis, como parte inicial de um estudo no contexto de projetos de novos produtos, sem a pretensão de se estabelecerem relações de causa e efeito, servindo de inspiração e contribuição para futuros trabalhos mais aprofundados.

É importante considerar também que todas as análises associativas aqui empreendidas foram feitas em termos globais, sem se considerar um infinito número de variáveis moderadoras que poderiam interferir na relação entre a orientação para mercado e a interface funcional. Certamente, as características

Revista de Administração e Inovação, São Paulo, v. 10, n.3, p.184-207, jul./set. 2013. 
dos setores econômico-industriais, das empresas, dos projetos e dos próprios indivíduos pesquisados seriam exemplos de variáveis de tal ordem, que poderiam conduzir a resultados diferenciados dos aqui apresentados. Esta, portanto, é outra limitação com que o presente estudo deve ser visto, estimulando que outros, nessa linha, sejam desenvolvidos no futuro.

Outro ponto importante a ser observado está em que os resultados não podem ser generalizados para todos os projetos de desenvolvimento de novos produtos, dada a limitação da amostra utilizada no estudo, composta intencionalmente e com apenas 21 respondentes, fatos não considerados estatisticamente representativos para projeções para o resto da população. Para que seja possível se aplicarem mais amplamente os entendimentos sobre a relação entre orientação para mercado e interface funcional, sugere-se que as próximas pesquisas a respeito do tema abranjam uma quantidade maior de pessoas entrevistadas e sigam um critério de amostragem que permita inferências estatísticas para a população do estudo.

Ainda como limitação, um último ponto deve ser relembrado: os resultados deste estudo são fruto da opinião dos entrevistados, o que também condiciona a precisão dos resultados encontrados. O fato de os questionários terem sido aplicados pela Internet, dentro do que se convenciona como $e$-survey, também contribuiu para que ocorressem possíveis vieses, advindos de interferências externas, que fogem ao controle do pesquisador (Vasconcellos e Guedes, 2007).

Embora típicos de estudos no campo das áreas sociais aplicadas, esses vieses não devem ser menosprezados. Uma forma de reduzi-los seria utilizar métodos de triangulação de respostas ou mesmo aplicar um reteste do questionário a uma parcela dos informantes, procedimento que poderia revelar pelo menos um índice de confiabilidade do instrumental. Tais mecanismos são fortemente sugeridos para outros estudos deste tipo.

Quanto a recomendações para as organizações, os resultados encontrados neste estudo sugerem que a captação e disseminação das informações provenientes do mercado, bem como a resposta dada a esses inputs podem estar diretamente relacionadas com a confiança despertada e a integração obtida entre os membros das equipes de projetos de desenvolvimento de novos produtos. Nesse sentido, entende-se que as organizações, ao iniciarem um projeto, devem ficar atentas ao tipo de informação captada no mercado. Do mesmo modo, devem utilizar mecanismos e ferramentas adequados de disseminação da informação, para que não haja distorções no processo, pois informações disseminadas de forma inadequada também podem abalar a integração e a confiança entre as pessoas. Além disso, devem buscar responder de forma adequada, e mais rapidamente possível, às informações geradas e disseminadas durante o ciclo dos projetos.

Revista de Administração e Inovação, São Paulo, v. 10, n.3, p.184-207, jul./set. 2013. 
Quanto às recomendações para estudos teóricos, sugere-se que este estudo, ou instrumento semelhante, seja aplicado a uma amostra mais representativa, para que algumas das proposições aqui surgidas sejam confirmadas estatisticamente. Sugere-se, ainda, que sejam desenvolvidos estudos correlacionais mais potentes, para se estabelecerem, mais precisamente, os graus de influência das variáveis independentes sobre variáveis independentes, com e sem a presença de variáveis moderadoras. Pode também ser feita a tentativa de análise contrária, considerando-se interface funcional como variável independente e orientação para mercado como variável dependente. De fato, é razoável supor-se que a existência de confiança e os mecanismos de integração utilizados pelas equipes de projetos também possam influenciar o desempenho da captação, disseminação e resposta à inteligência gerada com base nas informações de mercado. Por fim, sugere-se a realização de pesquisas qualitativas, em busca da compreensão das razões mais profundas para as relações entre orientação para mercado e interface funcional.

\section{REFERÊNCIAS}

Atuahene-Gima, K (1995). An exploratory analysis of the impact of market orientation on new product performance: a contingency approach. Journal of Product Innovation Management, 12, 275-293.

Bamber, D., Owens, J., Davies, J. \& Suleman, A. (2002). Enabling the emergent entrepreneurial organization to develop new products. International Journal of Entrepreneurial Behavior \& Research, 8 (4), 203-221.

Department for Business, Enterprise \& Regulatory Reform. (2008). The 2008 R\&D scoreboard. $\begin{array}{lllll}\text { Recuperado em } & 15 & \text { novembro } & 2010, & \text { de }\end{array}$ http://www.innovation.gov.uk/rd_scoreboard/downloads/2008_RD_Scoreboard_data.pdf

Bremser, W. G. \& Barsky, N. P. (2004). Utilizing balanced scorecard for R\&D performance measurement. R\&D Management, 34 (3), 229-238.

Brown , S. L. \& Eisenhardt, K. M. (1995). Product development: past research, present findings, and future directions. Academy of Management Review, 20 (2), 343-378.

Buss, C. O. (2002). Cooperação interfuncional no desenvolvimento de novos produtos: a interface marketing-engenharia. (2002). Dissertação de mestrado, Escola de Administração, Universidade Federal do Rio Grande do Sul, Porto Alegre, Brasil.

Calantone, R. J., Dröge, C. \& Vickery, S. (2002). Investigating the manufacturing-marketing interface in new product development: does context affect the strength of relationships? Journal of Operations Management, 20 (1), 273-287.

Revista de Administração e Inovação, São Paulo, v. 10, n.3, p.184-207, jul./set. 2013. 
Carvalho, M. M. \& Rabechini, R., Jr. (2008) Construindo competências para gerenciar projetos: teoria e casos. São Paulo: Atlas.

Cooper, R. G. (1995). How to launch a new product successfully. CMA, 69 (8), 20-23.

Cury, A. (2006). Organização e métodos: uma visão holística. São Paulo: Atlas.

Day, G.S. (1994). The capabilities of market-driven organizations. Journal of Marketing 58 (4), 37-52.

Deshpandé, R., Farley, J. U., \& Webster, F. E. (1993). Corporate culture, customer orientation, and innovativeness in Japanese firms: a quadrad analysis. Journal of Marketing, 57 (1), 23-27.

Diegel, O. (2002, fevereiro). Designing breakdown structures: an extension to the work breakdown structure to manage innovation in new product development projects. Proceedings of the Project Management Institute Annual Seminars \& Symposium, San Antonio, Texas, USA.

Dinsmore, P. C. Winning business with enterprise project management. (1998). New York: Amacom.

García, N., Sanzo, M. J. \& Trespalacios, J. A. (2008). New product internal performance and market performance: evidence from Spanish firms regarding the role of trust, interfunctional integration and innovation type. Technovation, 28 (11), 713-725.

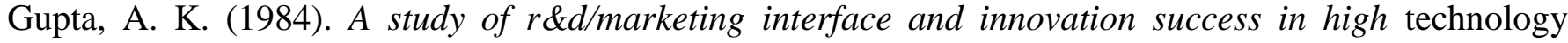
firms. Ph.D. dissertation, Syracuse University, New York, USA.

Hair, J., Anderson, R., Tatham, R. \& Black, W. (2008). Multivariate Data Analysis. Upper Saddle River: Prentice-Hall.

Kara, A., Spillan, J. E., \& Deshields, O. W, Jr. (2005). The effect of a market orientation on business performance: a study of small-sized service retailers using MARKOR Scale. Journal of Small Business Management, 43 (2), 105-118.

Kohli, A. K.; Jaworski, B. J. (1990). Market orientation: the construct, research propositions, and managerial implications. Journal of Marketing, 54 (4), 1-8.

Kohli, A. K.; Jaworski, B. J., \& Kumar, A. (1993). MARKOR: a measure of market orientation. Journal of Marketing Research, 30 (4), 467-477.

Kruglianskas, I. (1981). Efeito da interação organizacional na eficácia do centro de pesquisas e desenvolvimento cativo. Tese de Doutorado em Administração. Faculdade de Economia, Administração e Contabilidade da USP, São Paulo, Brasil.

Lima, M. O. (2010). Orientação para mercado e interface inter-funcional: um estudo no contexto do desenvolvimento de novos produtos. Dissertação de Mestrado em Administração, Faculdade de Economia, Administração e Contabilidade da USP, São Paulo, Brasil.

Littler, D. (1994). Marketing and innovation. In Dodgson, M. \& Rothwell, R. (Ed.). The handbook of industrial innovation. Cheltenham, UK: Edward Elgar.

Martins, A. P., Martins, M. R. Pereira, M. M. M, \& Martins, V. A. (2005). Implantação e consolidação de escritório de gerenciamento de projetos: um estudo de caso. Revista Produção, 15, (3), 404-415.

Revista de Administração e Inovação, São Paulo, v. 10, n.3, p.184-207, jul./set. 2013. 
Matsuno, K., Mentzer, J. T., \& Rentz, J. O. (2000). A Refinement and validation of the MARKOR scale. Journal of the Academy of Marketing Science , 28, (4), 527- 539.

Moenaert, R., \& Souder, W. (1990a). An information transfer model for integrating marketing and R\&D personnel in new product development projects. Journal of Product Innovation Management, 7, (1), 91107.

Moenaert, R., Souder, W., De Meyer, A., \& Deschoolmeester, D. (1994). R\&D - marketing integration mechanisms, communication flows, and innovation success. Journal of Product Innovation Management, $11(1), 31-45$.

Müller, H. F., Neto. (2005). Inovação orientada para o mercado: um estudo da relação entre orientação para mercado, inovação e performance. Tese de Doutorado em Administração, Escola de Administração da UFRGS, Porto Alegre, Brasil.

Narver, J. C., \& Slater, S. F. (1990). The effect of a market orientation on business profitability. Journal of Marketing, 54, (10), 20-35.

Neely, A., \& Hii, J. Innovation and business performance. (1998). The Judge Institute of Management Studies, University of Cambridge, 1-49.

Organization for Economic Co-operation and Development. Oslo manual: proposed guidelines for vollecting and interpreting technological innovation data. Recuperado em 15 novembro, 2010, de http://www.oecd.org/document/23/0,3343,en_2649_34409_35595607_1_1_1_1,00.html.

Purdon, W. A. B. (1996). Increasing R\&D effectiveness: researches as business people. Research Technology Management, 39 (4), 48-56.

Rabechini Jr., R., Carvalho, M. M., \& Laurindo, F. J. B. (2002). Fatores críticos para implementação de gerenciamento por projetos: o caso de uma organização de pesquisa. Revista produção, 12 (2), 28-41.

Rodrigues, I., Rabechini, R. R., Jr., \& Csillag, J. M. (2006). Os escritórios de projetos como indutores de maturidade em gestão de projetos. $R$. Adm., 41 (3), 273-287.

Roussel, P. A., Saad, K. N., Bohlin, N. (1992). Pesquisa \& Desenvolvimento: como integrar P\&D ao plano estratégico e operacional das empresas como fator de produtividade e competividade. São Paulo: Makron Books.

Schumpeter, J. A. (1982). A teoria do desenvolvimento econômico. São Paulo: Editora Abril.

Sampieri, R., Collado, C., \& Lucio, P. (2006). Metodologia da pesquisa. São Paulo: McGraw-Hill.

Shapiro, B.P. (1977). Can marketing and manufacturing coexist? Harvard Business Review, 55 (5), $104-$ 114. (1988). What hell is market oriented? Harvard Business Review, 55 (5), 119-125.

Siegel, S. (2006). Estatística não paramétrica para ciências do comportamento. Porto Alegre: Artmed.

Revista de Administração e Inovação, São Paulo, v. 10, n.3, p.184-207, jul./set. 2013. 
Slater, S. F., \& Narver, J. C. (1994). Market orientation, customer value and superior performance. Business Horizon, 37 (2), 22-28.

Song, X. M., \& Parry, M. E. (1997). The determinants of Japanese new product success. Journal of Marketing Research, 34 (1), 64-76.

Song, X. M., \& Thieme, R. J. (2006). A cross-national investigation of the R\&D-marketing interface in the product innovation process. Industrial Marketing Management, 35 (3), 308-322.

Tajeddini, K., Trueman, M., \& Larsen, G. (2006). Examining the effect of market orientation on innovativeness. Journal of Marketing Management, 22 (5/6), 529-551.

Vasconcellos, L., \& Guedes, L. F. A. (2007). E-Surveys: Vantagens e Limitações dos Questionários Eletrônicos via Internet no Contexto da Pesquisa Científica. Anais do Encontro Nacional da Associação Nacional de Pós-Graduação e Pesquisa em Administração, Rio de Janeiro, RJ, Brasil.

Vasquez, R., Santos, M. L., \& Álvares, L. I. (2001). Market orientation, innovation and competitive strategies in industrial firms. Journal of Strategic Marketing, 9 (1), 69-90.

Verhees, F. (2005). Market oriented product innovation in small firms. Tese de Doutorado, Wageningen University, Alemanha.

Zawislak, P. A. (1995). A relação entre conhecimento e desenvolvimento: essência do progresso técnico. Análise, 6 (1), 125-149. 


\title{
MARKET ORIENTATION AND FUNCTIONAL INTERFACE: EVIDENCE IN DEVELOPMENT PROJECTS OF NEW PRODUCTS
}

\begin{abstract}
Innovation has been recognized as very important to firm growing and competition. One key-factor for innovation success is to transform market signals in adequate answers in a form of new/improved products and services introduced into the market. In this sense, the main purpose of this paper is to examine if firms with a better functional integration respond more adequately to market need signals. The paper initially presents an extensive literature review on this subject, extracting the main premises, concepts and appropriated terminology, serving as study background. The data then was collected trough an e-survey, involving 21 respondents from different and innovative firms in Brazil, intentionally chosen. The utilized questionnaire was pre-tested and validated using the Crombach alpha coefficient and all respondents were significantly involved in product development efforts in those firms. For data analysis, non-parametric statistics (rho, from Spearman) were employed. Taking to the perceptions of the respondents, the results demonstrated firstly that the majority of firms tend to present moderated or high degrees of market orientation and functional interface. Secondly, the correlations among several indicators found in the main portion of the sample also indicated that firms more oriented to the market also showed better integration and confidence between functional areas involved in product development activities. Those results, however, due to the main limitations presented, need more in depth studies to be generally accepted.
\end{abstract}

Keywords: Innovation, management, functional interface, projects, product development.

Data do recebimento do artigo: 05/03/2013

Data do aceite de publicação: 18/07/2013

Revista de Administração e Inovação, São Paulo, v. 10, n.3, p.184-207, jul./set. 2013. 